\title{
The development of an acute frailty team
}

\author{
Authors: Scott Mather ${ }^{*}$ and Marie Hanley
}

\section{Introduction}

Older people living with frailty are more likely to be admitted to hospital when attending the emergency department (ED). Following admission, this population is also more likely to have prolonged inpatient stay, patient safety incidents and higher inpatient mortality. ${ }^{1}$ NHS Improvement recommends that all patients aged over 65 attending the ED should be screened for frailty using a validated screening tool such as such as the Clinical Frailty Scale (CFS). ${ }^{2}$ In addition, all patients with frailty should have access to a team capable of assessing frailty syndromes within the ED.

Manchester Royal Infirmary, part of Manchester University NHS Foundation Trust, established a frailty project group to meet these objectives. My role as an Royal College of Physicians chief registrar has allowed me to help lead the group and design improvement projects.

\section{Intervention}

One of these projects involved the pilot of an acute frailty team (AFT) within the ED, comprising a consultant geriatrician, nurse consultant and advanced nurse practitioner. Further support was provided from the ED pharmacist and Discharge 2 Assess (D2A). Older patients ( $\geq 65$ ) presenting to the ED with a CFS of $\geq 4$ were reviewed by the frailty team (weekdays, $9 \mathrm{am}-4 \mathrm{pm}$ ) providing they did not meet the pilot's exclusion criteria (such as trauma, stroke or medical emergency). Patients were identified by referral or case finding. These patients then underwent a Comprehensive Geriatric Assessment within the ED.

\section{Results}

During the pilot 83 patients were reviewed over 23 days from October-November 2018. Only 39\% of patients reviewed by the AFT were admitted to hospital. The average CFS score was 5.2 and the average time for review was 55 minutes. The presenting complaint was variable, however $40 \%$ of patients presented following a fall. Of those admitted, $47 \%$ were discharged within 48 hours compared with $40 \%$ of patients who were not reviewed by AFT. Prior to review the majority ( $55 \%$ ) had already been referred to the medical team for admission. The 28 day re-attendance rate was $22 \%$. The key limiting factors identified which prevented discharge were delays for therapy assessment and medications.

Authors: Manchester University NHS Foundation Trust *RCP chief registrar

\section{Conclusions}

This pilot highlighted that an AFT within the ED can safely discharge patients or commence management which will reduce a patient's subsequent length of stay. As part of the frailty project group, this data is now forming the basis of the case for the development of a sustainable AFT. Furthermore, limitations identified demonstrated the need to widen the multidisciplinary nature of the team to increase effectiveness. Further pilots are planned following the implementation of ED frailty screening and development of a partnership with primary care.

\section{References}

1 Gregorevic KJ, Hubbard RE, Lim WK, Katz B. The clinical frailty scale predicts functional decline and mortality when used by junior medical staff: a prospective cohort study. BMC Geriatr 2016;16:117.

2 NHS Improvement. Ambulatory emergency care guide: same day acute frailty services. NHS Improvement, 2018. 